\title{
Mechanism of pitting corrosion protection of metals and alloys in new-generation water treatment plants
}

\author{
Vladimir Grachev ${ }^{1,}$, , Igor Nechaev ${ }^{2}$, Andrei E. Rozen ${ }^{3}$, and Andrei A. Rozen ${ }^{3}$ \\ ${ }^{I}$ Rosatom State Corporation, 24 Bolshaya Ordynka Str., 119017 Moscow, Russian Federation \\ ${ }^{2}$ «Karpov Institute of Physical Chemistry», 10-bld. 1 Vorontsovo Pole str., 105064 Moscow, Russian Federation \\ ${ }^{3}$ Penza State University, 40 Krasnaya St., 440026 Penza, Russian Federation
}

\begin{abstract}
In this article authors set out a principle of pitting corrosion protection, suggested a new class of multilayer materials with high corrosion resistance. They substantiated the choice of the layers for the multilayer material designed for exploitation in oxidizing and non-oxidizing environment. The sphere of application of the multilyer materials was defined.
\end{abstract}

\section{Introduction}

Currently, annual direct metal losses resulting from corrosion make up $12 \%$ of the total weight of metal in stock in Russia, which corresponds to the loss of up to $30 \%$ of annually produced metal1. Global corrosion losses account for $4-6 \%$ of the national income in developed countries [2]. Especially strongly this process is expressed in water-supply systems. Which historically, for a long while, have been principally made of steel pipes. At an average service life of water conduits and distribution network over 30 years, most part of metal conduits are in the sad state. Indirect losses associated with the structural failure, process shutdown and depressurization of equipment cause even greater economic damage. This leads to the necessity of improving the methods of protection against corrosion and developing new corrosion resistant materials and products.

Modern chemical, petrochemical and oil refining industries, as well as communal service branches, are characterized by engineering units of high capacity and processing medium that is highly corrosive. This leads to the use of equipment with an increased metal content. According to the estimates made by Japanese experts [3] and data provided by the NACE International (International Association of Corrosion Engineers) [4], more than $60 \%$ of the engineering units' failures occur due to the influence of temperature and corrosive environment, and the annual cost of corrosion is estimated at \$2.2-2.5 trillion. In particular, in 2014, they amounted to about $\$ 445.0$ billion in the USA, $\$ 95.0$ billion - in Germany, more than $\$ 50.0$ billion - in Russia. This corresponds to the loss of up to $30 \%$ of the annually produced metal.

\section{Matherials and methods}

When designing a new unit, it is important to use materials with an improved corrosion resistance, e.g. multilayer materials with an internal protector $[5,8,9]$. This solution is particularly true for the industrial water treatment systems as well as for water carriage systems of the big industrial units. Namely in such systems, especially difficult environment for pipelines and metal facilities service, is observed. At the same time, there has not been any accomplished technical solution that would make it possible to use the internal protector in order to increase the guaranteed technical lifetime of the products operating in the conditions of an increased corrosion attack.

Pitting corrosion is typical for metals susceptible to passivation due to the presence of strong oxidants or external anodic protection. The main condition for the pitting occurrence is an electrochemical potential shift towards more a positive one than a certain critical value, which is known as a pitting potential. Simultaneous presence of activating anions of fluorine, chlorine, bromine, iodine, and of such oxidizing agents as oxygen in the solution favors pitting formation. Superficially, active haloid ions are adsorbed on the metal surface and displace oxygen that ensures a passive state. Oxide film destruction takes place and the surface activation occurs. Pitting formation may happen at the locations with a distorted structure, non-metallic inclusions, and reduced concentration of chromium and other alloying elements in steel. The surface of pitting becomes anodic, and the rest of the passive metal surface becomes cathodic.

The difference in the activation potential and the steady-state (equilibrium) potential is regarded as a characteristic of resistance to pitting corrosion. The larger the difference, the more resistant to pitting corrosion the metal is.

\footnotetext{
* Corresponding author: secretary@nifhi.ru
} 
In this regard, technical solution for producing a multilayer material with the internal protector is of a certain interest.

The corrosion process is transformed due to the multilayer structure. Pitting corrosion of the outer layer shifts to the contact corrosion of the specific sacrificial layer (Fig.1).

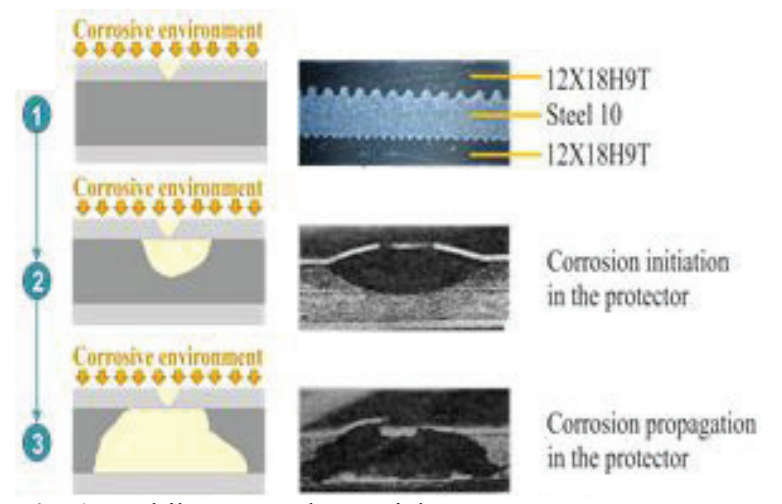

Fig. 1. Multilayer metal material.

The key point of the suggested technology is as follows. Instead of mono- or bimetal, a multilayer material with at least three layers is used. Such multilayer composite can be adapted for the one-way or two-way contact with the corrosive environment or media of a different composition. Composition of the multilayer material's layers depends on the composition of media and on the electrochemical potentials of the constituent metals. Applying a protector between the protected layers is principally new.

We have considered two cases:

- When a multilayer material contacts with the processing medium containing aqueous solutions of alkalis, acid salts or acids, whose anions are not oxidizing agents;

- When a multilayer material contacts with the processing medium containing aqueous solutions of alkalis, acid salts or acids, whose anions are oxidizing agents. It allows of further discussion of the first and second type multilayer composite

\subsection{Multilayer composite of the first type}

For the first (outer) layer contacting the processing nonoxidizing medium, a material that has a sufficiently high corrosion resistance and passivity in such environment should be selected.

The material of the first layer is characterized by the anode A curve and the cathode K curve (Fig.2). There is an area of passivity on the anode curve. When the layer contacts with the corrosive environment that does not contain oxidants, a fixed potential $\mathrm{E}_{1}$ is set on the layer.

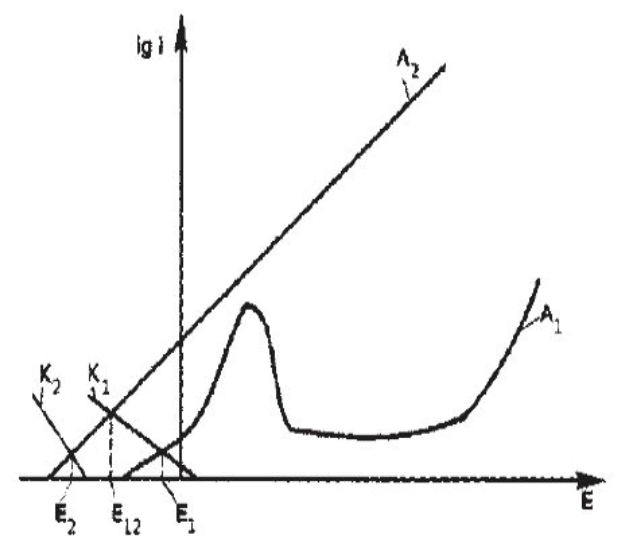

Fig. 2. Schematic arrangement of the metal polarization curves in the multilayer material: $K_{1}, K_{2}$ - cathodic curves of the outer and inner layers; $A_{1}, A_{2}$ - anodic curves, respectively.

As a result of the medium action, corrosion spots in the form of pitting appear in the outer layer. These corrosion spots deepen over time and penetrate into the second layer. The material of the second layer is selected so that the value of its steady-state electrochemical potential E2 in contact with the processing medium is lower than the steady-state electrochemical potential of the first metal layer. The state of the second layer material - the protector - is characterized by the anode A2 and cathode $\mathrm{K} 2$ curves.

When pitting reaches the second layer metal, the steady-state potential E12 is set due to the contact potential difference of the first and second metal layers. When this happens, the second layer metal becomes the anode, and the first layer metal becomes the cathode. The second layer becomes the protector, i.e. a sacrificial electrode, and gradually dissolves. Anodic dissolution reaction can proceed until the formation of a sizable cavity - a lens - in the protector. On the first layer material, depending on the composition of the medium, the evolution of hydrogen, the reduction of oxygen or other electrochemical reactions take place.

Anode:

$$
\mathrm{Fe} \rightarrow \mathrm{Fe}+2+2 \mathrm{e}
$$

Cathode:

$$
\begin{gathered}
2 \mathrm{H}++2 \mathrm{e} \rightarrow \mathrm{H} 2 \\
\mathrm{Fe}+3+\mathrm{e} \rightarrow \mathrm{Fe}+2
\end{gathered}
$$

The Composition of the third layer is similar to the first one. When the depth of the cavity in the protector becomes equal to its thickness, the third layer also becomes the cathode - the same as the first layer. The corrosion rate of the second layer may increase, and then corrosion may take place until the complete dissolution of the protector. If the reaction products are insoluble materials, they can slag individual pitting sites and reduce the rate of destruction of the three-layer material as a whole.

Fig.3 represents the corrosion dynamics in the multilayer material in the medium containing aqueous 
solutions of alkalis, acid salts or acids, whose anions are not oxidizing agents.

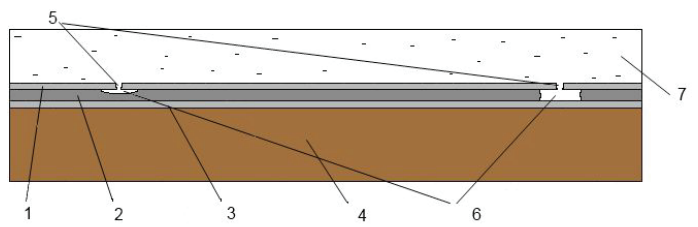

Fig. 3. Corrosion dynamics in the multilayer material in the medium that doesn't contain oxidizing agents: 1 - outer layer in contact with the processing medium; 2 - protector; 3 - third layer; 4 - base layer; 5 - pitting in the outer layer; 6 - lens in the protector; 7 - corrosive environment

\subsection{Multilayer composite of the second type}

For the first (outer) layer contacting the processing oxidizing medium, a material that has a sufficiently high corrosion resistance and passivity in such environment should be selected

The second layer's steady-state electrochemical potential should be higher than the first layer electrochemical potential; moreover, the material of the second layer should have lower hydrogen overvoltage than the material of the outer layer. The first layer material is characterized by the anode A1 and cathode $\mathrm{K} 1$ curves (Fig.4). There is an area of passivity on the anode curve.

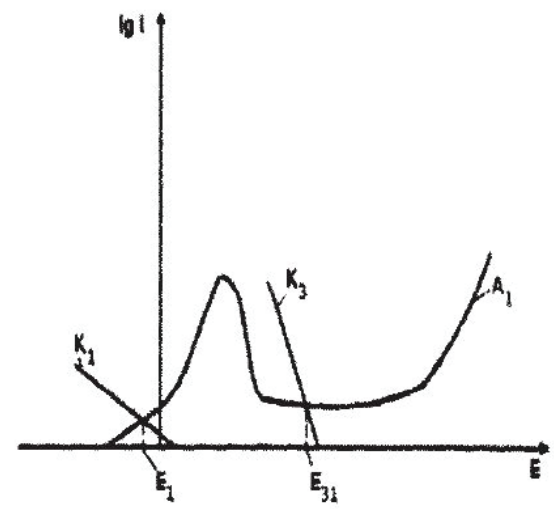

Fig. 4. Schematic arrangement of the metal polarization curves in the multilayer material: $\mathrm{K}_{1}$ - cathodic curve of the outer layer; $\mathrm{A}_{1}$ - anodic curve of the outer layer; $\mathrm{K}_{3}$ - cathodic curve of the inner layer.

While in operation, pitting is formed in the outer layer and penetrates up to the second layer in the course of time. The contact potential difference between the layers appears. The second layer metal retains its passive state. Anodic dissolution of the first layer metal is inhibited due to the formation of the reaction products that are slightly soluble. The potential of the first layer metal shifts to more positive values of E31 potentials; it leads to additional passivation and, as a result, stops the growth of pitting.

In this case, the corrosion potential of the outer layer steel maintains a stable positive value corresponding to the passive state. While in operation, the passive film on the outer layer may dissolve due to the chemical reaction.

Fig.5 represents the corrosion dynamics in the multilayer material in the medium containing aqueous solutions of alkalis, acid salts or acids, whose anions are oxidizing agents.

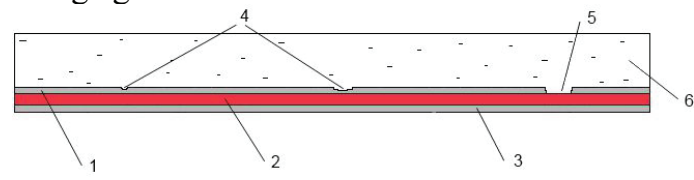

Fig. 5. Corrosion dynamics in the multilayer material in the medium that contain oxidizing agents: 1 - outer layer in contact with the processing medium; 2 - protector; 3 - third layer; 4 - pitting in the outer layer; 5 -dissolution of the outer layer; 6 - corrosive environment.

Composition of the third layer material is identical to the material of the first outer layer.

Table 1 shows structural metals and alloys in the order of increasing Ecor corrosion potential in sea water $[6,10,11]$. Data in the table indicate that the grades 10 , 15,20 low-carbon steels have a more negative potential compared with the $12 \mathrm{X} 17,12 \mathrm{X} 18 \mathrm{H} 9,10 \mathrm{X} 17 \mathrm{H} 13 \mathrm{M} 2 \mathrm{~T}$ high-alloy steels in the passive state. It means that lowcarbon steels may be used as a protector in combination with the corrosion-resistant high-alloy steels in the media containing aqueous solutions of alkalis, acid salts or acids, whose anions are not oxidizing agents

According to the Fig. 5, the 12X17, 12X18H9, $10 \mathrm{X} 17 \mathrm{H} 13 \mathrm{M} 2 \mathrm{~T}$ high-alloy steels in active state have a more negative potential compared with copper, titanium, and Hastelloy. It means that high-alloy steels can be used as a protector in combination with the indicated metals and alloys in media containing aqueous solutions of alkalis, acid salts or acids, whose anions are oxidizing agents.

\begin{tabular}{|c|c|c|c|}
\hline Metal & $E_{\text {cor }}$ B & Metal & $E_{\text {cor }}$ B \\
\hline Magnesium & -1.45 & Brass $(40 \mathrm{Zn})$ & -0.20 \\
\hline Magnesium alloy $(6 \mathrm{~A} 1,3 \mathrm{Zn}, 0,5 \mathrm{Mn})$ & -1.20 & Bronze $(6 \ln )$ & -0.20 \\
\hline Zinc & -0.80 & Nickel (active state) & -0.12 \\
\hline Aluminum alloy (10 Mg) & -0.74 & Brass $(30 \mathrm{Zn})$ & -0.10 \\
\hline Aluminum alloy $(10 \mathrm{Zn})$ & -0.70 & Bronze (5-10 A 1$)$ & -0.10 \\
\hline Aluminum & -0.53 & Copper & -0.08 \\
\hline Duralumin & -0.50 & $\begin{array}{l}\text { Corrosion-resistant steel } 20 \times 13 \\
\text { (passive state) }\end{array}$ & +0.03 \\
\hline Iron & -0.50 & Nickel (passive state) & +0.05 \\
\hline Carbon steel & -0.40 & $\begin{array}{l}\text { Corrosion-resistant steel } \\
12 \times 17 \text { (passive state) }\end{array}$ & +0.10 \\
\hline Gray cast-iron & -0.36 & $\begin{array}{l}\text { Corrosion-resistant steel 12X18H9 } \\
\text { (passive state) }\end{array}$ & +0.17 \\
\hline $\begin{array}{l}\text { Corrosion-resistant steel } \\
12 \mathrm{X} 13 \text { and } 12 \mathrm{X} 17 \text { (active state) }\end{array}$ & -0.32 & Hastelloy (Ni, $20 \mathrm{Mo}, 18 \mathrm{Cr}, 6 \mathrm{~W}, 7 \mathrm{Fe}$ ) & +0.17 \\
\hline $\begin{array}{l}\text { Corrosion-resistant steel } \\
\text { 12X18H9 (active state) }\end{array}$ & -0.30 & $\begin{array}{l}\text { Corrosion-resistant steel } \\
\text { 10X17H13M2T (passive state) }\end{array}$ & +0.20 \\
\hline $\begin{array}{l}\text { Corrosion-resistant steel } \\
\text { 10X17H13M2T (active state) }\end{array}$ & -0.30 & Silver & $\begin{array}{l}+0.12 \ldots \\
+0.20\end{array}$ \\
\hline Lead & -0.30 & Titanium & $\begin{array}{l}+0.12 \ldots \\
+0.20\end{array}$ \\
\hline
\end{tabular}

Fig.5. Ecor potential values in sea water

Any multilayer materials under development should have at least three layers $[12,14]$. A three-layer material should be composed by matching materials for each layer so that the corrosion of the product is minimal, whereas the protective ability is maximal. As the base layer, a layer of structural steel of the required thickness can be welded to the multilayer material in order to achieve high structural strength. 
Materials containing two or more layers of different composition may be obtained by various technologies. These include cast sheathing, hot pack rolling, cold sheathing, explosive welding, and weld deposition [7, $13,14,15,18]$. One of the most popular and highly efficient methods of industrial production of multilayer materials is explosive welding.

Explosive welding is a high-velocity welding under pressure, wherein a non-detachable joint is formed as a result of the welded elements'collision.

The source of energy is explosives. Upon explosive initiation, a detonation front (shockwave) spreads, and an expansion of gaseous products occurs. There is a jump in pressure, which enables high acceleration of the element colliding with the stationary element. In the collision area, pressure can reach $10 \ldots 20 \mathrm{GPa}$. There is a joint plastic deformation of the metals in a relatively thin layer that is up to $2 \mathrm{~mm}$ deep. The result is a firm nondetachable joint.

Explosive welding has advantages due to short duration and high pressure, which is achieved in the detonation of the explosives $[17,19,20]$. These advantages can be pointed out as follows:

1. It is possible to obtain compounds of similar and dissimilar metals and alloys, including those with differing physical and mechanical properties (melting point, coefficient of linear thermal expansion, strength, ductility).

2. Chemical composition of the base and cladding layers doesn't change during the explosive welding, and mechanical properties vary in a narrow band of width of $3 . . .6 \mathrm{~mm}$ that is adjacent to the joint line.

3. Explosive welding is carried out in the solid phase; this allows joining materials that form brittle intermetallic compounds.

4. It is possible to obtain compounds with the area of $20 \mathrm{~m} 2$ and more, the thickness of the base layer that is limited only by the sheet or product gauge, and the cladding layer thickness from fractions of a millimeter to several millimeters.

5. It is possible to obtain multilayer materials in a single welding cycle.

6. There is no need for complicated and expensive equipment.

7. The cost of consumables and energy consumption is relatively low.

8. It is possible to produce flat blanks and cylindrical billets and to clad curved surfaces.

According to the Skolkovo Innovation Center, the global market of produced corrosion-resistant multilayer material in various industries amounts to more than $\$ 900$ million. Of these, $33 \%$ is energy industry, $25 \%-$ oil refining industry, $21 \%$ - chemical industry, $14 \%$ nuclear industry, and 7\% - shipbuilding

\section{Conclusion}

Depending on the corrosive environment, the equipment's operational life is increased by a factor of 515 as compared with austenitic stainless steels. A multilayer metal material consists of minimum number of expensive components. It is possible to carry out costneutral and safe external diagnostics of corrosion, including ultrasonic testing.

The obtained multilayer metal material is a completely new product. There are no direct equivalents. The main areas of application are chemical, oil and gas industries and nuclear power. The obtained material is in the same segment with tantalum and platinum by performance characteristics, and it is at the same segment with conventional chromium-nickel stainless steels by price range.

The present research was supported by the Ministry of Education and Science of the Russian Federation (Grant of the 20th of October, 2014, № 14.576.21.0053; The Applied Research Unique Identifier RFMEFI57614X0053)

\section{References}

1. E.N. Kablov, Science and Life, 11, 16-21, (2012)

2. V.N. Ivanovsky, Engineering practice, 6, 4-14, (2010)

3. ESPEC Technology Report. Test Navi Report No. 21 (Issue 78)

4. NACE International. Corrosion Resistant Coatings, (2011)

5. Multilayer material with enhanced corrosion resistance (embodiments) and method for its production (embodiments). EAPO patent $016878 \mathrm{dd}$ 30.08.2012.

6. N. D. Tomashov, G. P. Chernova, Metallurgiya, Moscow (1986)

7. A. G. Kobelev et al., Intermet Engineering, Moscow, (2002)

8. Yu. A. Konon, L. B. Pervukhin, A. D. Chudnovskii, Mashinostroenie, Moscow, (1987)

9. A. E. Rozen, I. S. Los, Yu. P. Perelygin, L. B. Pervukhin, Yu. A. Gordopolov, G. V. Kiriy, P. I. Abramov, S. G. Usatyi, D. B. Kryukov, O. L. Pervukhina, I. V. Denisov, A. A. Rozen. Eurasian Patent 016878, (2008)

10. B. Wallén, S. Henrikson, Werkstoffe und Korrosion, 40, 602, (1989).

11. A. Mollica et al, Corrosion, 45, 1, 48-56, (1989)

12. A. E. Rozen, L. I. Reformatskaya, E. V. Kuznetsov, I. S. Los, P. A. Abramov, I. V. Denisov, A. V. Khorin, A. A. Rozen, Explosive Production of New Material: Science, Technology, Business, and Innovations, 9, (2010).

13. S. Yu. Kireyev, I. S. Los, A. E. Rozen, Yu. P. Perelygin, G. V. Kozlov, D. B. Kryukov, A. A. Rozen, Explosive Production of New Materials: Science, Technology, Business, and Innovations, 68, (2012).

14. I. S. Los, A. E. Rozen, Yu. P. Perelygin, S. G. Usatyi, A. V. Khorin, Resource-Saving Technologies to Repair, Restore, and Reinforce Parts of Machines, 
Mechanisms, Equipment, Tools, and Accessories from Nano- to Macrosize, 1, 465-468, (2010).

15. Yu. P. Perelygin, S. Yu. Kireyev, I. S. Los, A. E. Rozen, Prot. Met. Phys. Chem. Surf., 60 (7), 856859 (2014).

16. H. Bisadi, M. Khaleghi Meubodim J. Mech. Eng. Sci., 225, (2011).

17. F. Findik, Mater Des, 32, 1081-1093, (2011)

18. S. Saravanan, K. Raghukandan, Materials and Manufacturing Processes, (2013).

19. J. Song, A. Kostka, M. Veehmayer, D. Raabe, Mat Sci Eng A, (2011).

20. H. Paul, M. Faryna, M. Prazmowski, R. Banski, Arch. Metall. Mater., 56, 463-474 (2011). 